\title{
CAMLOG
}

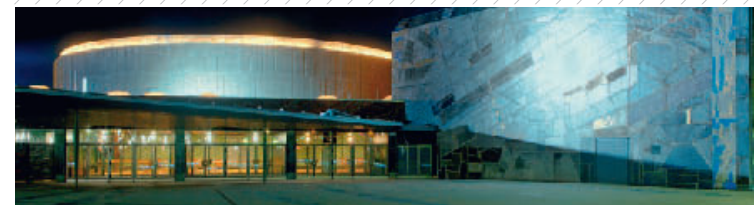

\section{CAMLOG Kongress in Stuttgart}

Die ersten Broschüren waren noch nicht gedruckt, da trafen bei CAMLOG bereits Online-Anmeldungen im zweistelligen Bereich ein. Offensichtlich sind das Interesse und die Erwartungen in Hinblick auf den 3. Internationalen CAMLOG Kongress vom 10. bis 12 . Juni 2010 in Stuttgart enorm hoch - analog zu den überdurchschnittlichen Maßstäben, die CAMLOG selbst an die Vielfältigkeit und Güte des Kongressprogramms und die Qualifikation der TopReferenten setzt.

Dies zeigt erneut klar die Firmenziele: wissenschaftlich fundierte, aktuelle Fortbildung zu bieten, neue Benchmarks in der Branche zu setzen und den Anwendernutzen ständig weiter zu erhöhen. Die renommierten Namen des wissenschaftlichen Kongress-Komitees sprechen für sich und bürgen für Qualität: Prof. Jürgen Becker, Dr. Sven Marcus Beschnidt, Prof. Rolf Ewers, Prof. Robert Sader, PD Dr. Frank Schwarz, Prof. Wilfried Wagner.

Auch wenn die Mehrzahl der Referenten aus Deutschland stammt, die Internationalisierung nimmt eine immer gewichtigere Rolle für die CAMLOG Gruppe ein. Beleg dafür ist die ständig wachsende Zahl international renommierter Kongress-Referenten.

CAMLOG erwartet für den Kongress in Stuttgart eine deutliche Erhöhung der Teilnehmerzahl im Vergleich zu den vergangenen Kongressen, die mit ca. 700 Teilnehmern schon sehr gut besucht waren. Die Kongressgebühr beträgt € 490.-; optional können attraktive Workshops, die am Vortag des zweitägigen wissenschaftlichen Programms stattfinden, gebucht werden.

$\boldsymbol{\lambda}$ Weitere Informationen zum Kongress und das Anmeldeformular finden Sie unter

Tel. 07044 - 9445-603

www.camlogcongress.com

$\mathrm{KaVo}$

\section{Artikulatorprogrammierung und Funktionsdiagnostik live am Patienten}

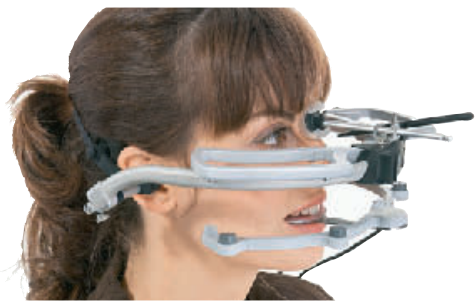

Lange haben die Anwender und Interessenten des KaVo ARCUSdigma 3D Messsystems warten müssen: Jetzt ist endlich eine DVD verfügbar, die das innovative System anhand einer Live-Behand- lung am Patienten ausführlich darstellt und die umfangreichen Einsatzmöglichkeiten in der täglichen Praxis aufzeigt.

Insgesamt stehen dem Anwender mit dem System bis zu neun verschiedene Messprogramme für Therapie und Diagnostik zur Verfügung. Um jederzeit zu allen Programmen optimale Informationen zu erhalten, werden alle relevanten Behandlungsschritte am Patienten und weiterführend im zahntechnischen Labor mit Hilfe der neuen DVD demonstriert. Zahlreiche verschiedene Anwendungsgebiete können auf der DVD gezielt ausgewählt werden. Ob zum Beispiel individuelle
Artikulatorprogrammierung, Bestimmung der zentrischen Unterkieferposition, Schienentherapie, Bewegungsanalyse oder Lokalisation einer schmerzhaften Kiefergelenksposition mit automatischer Schienenberechnung: Der Anwender erhält alle gewünschten Informationen in ansprechend visualisierter Form.

Erhältlich ist die DVD ab sofort direkt über KaVo (Bestell.-Nr. 1.003.9354).

入 Tel. 07351 - 56-1599

info@kavo.com

www.kavo.com
Komet/Gebr. Brasseler

\section{Spitzen Antrieb}

Komet denkt immer "mit System". Es ist bei dem Angebot an Schallspitzen nur eine logische Konsequenz, auch ein passendes Schallhandstück ins Programm mit aufzunehmen: das SF1LM. Es ergänzt das professionelle Sortiment.

Schallhandstücke sind grundsätzlich luftbetrieben - so auch dieses. Es wird einfach auf den Turbinenschlauch der Behandlungseinheit gesteckt und passt auf alle MULTIflex ${ }^{\oplus}$-Kupplungen - übrigens die gängigste Variante im Markt.
Zwei gebündelte Lichtaugen leuchten das Arbeitsfeld optimal aus und erlauben ein sehr effizientes Vorgehen bei einer Schwingfrequenz von $6.000 \mathrm{~Hz}$. Der Leistungsregler lässt sich stufenlos einstellen.

Mit dem Spüladapter SF1978 ermöglicht Komet die validierte Aufbereitung der Schallspitzen in Miele Thermodesinfektoren. Eine schlaue Maßnahme, die erkennen lässt, wie stark sich das Unternehmen für Sicherheit, Ökonomie und Qualität macht. Das unterstreicht nicht zuletzt die zwei Jahre Herstellergarantie, die Komet gibt.

入 Tel. 05261 - 701-700 info@brasseler.de www.kometdental.de 